\title{
Long Period Variables as tracers of Galactic Structure
}

\author{
Martin A. T. Groenewegen \\ Royal Observatory of Belgium, Ringlaan 3, B-1180 Brussels, Belgium \\ email: marting@oma.be.
}

\begin{abstract}
Distances to a large sample of long period variables are derived using a PL-relation and a 3D model for the reddening. Their use as a tracer of galactic structure is discussed.
\end{abstract}

Almost all stars with initial masses $\lesssim 8 \mathrm{M}_{\odot}$ will pass through the Asymptotic Giant Branch phase. One of the main characteristics of this phase is radial pulsation as Irregular (Irr), semi-regular (SR) or Mira variables. Many of the SR actually show well behaved light-curves, and so these stars and the Miras are called Long Period Variables (LPVs).

That Mira variables follow a period-luminosity (PL-) relation had been known for decades, but in the optical the scatter is large. The practical use increased with the extension to the $K$-band or bolometric magnitude, especially with sufficient phase coverage of the light-curve. Recently Whitelock et al. (2008) revisited the PL-relations in $M_{\mathrm{bol}}$ and $M_{\mathrm{K}}$ for Miras, including the Galactic $P L$-relation using the 2007 revised Hipparcos parallaxes and other observables as constraints. With the $P L$-relation as distance indicator one can make an attempt to study Galactic Structure using LPVs, see Figure 1.

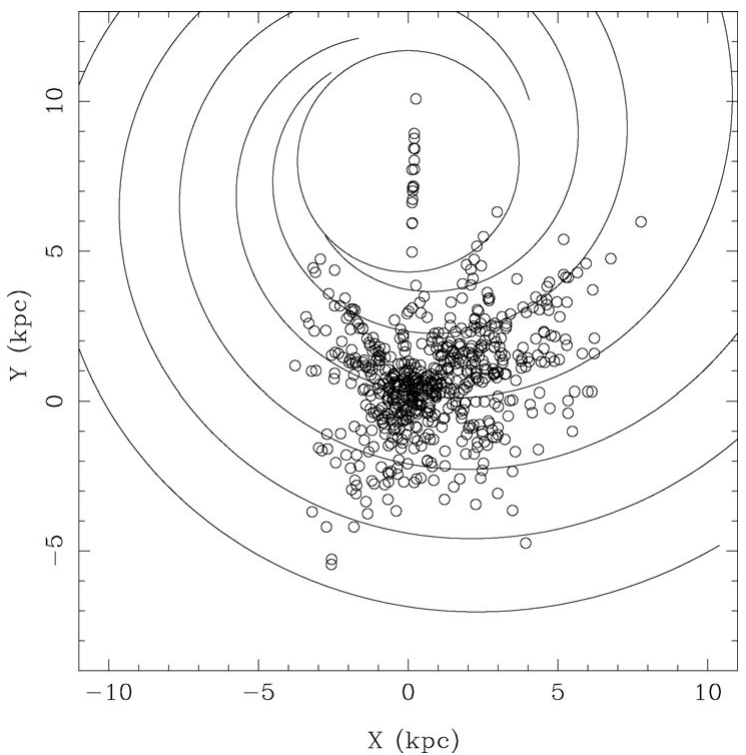

Figure 1. Location of $700 \mathrm{Mi}-$ ras, projected onto the Galactic Plane, with $|z|<500$ pc. The Hou 4-spiral arm model is shown as reference. From the GCVS 4500 stars classified as Miras were selected, with periods in the range $50-400$ d. Their $K$-band magnitudes were looked-up via SIMBAD (available for 1250). Using a PL-relation and a 3D model for the reddening (Groenewegen 2008) the distances were derived. I leave it up to the reader whether he/she believes that Miras trace Galactic Structure or not! The GCVS is clearly a very inhomogeneous source of information, so surveys like LSST and Skymapper will be essential in creating unbiased samples of LPVs.

\section{References}

Whitelock, P. A., Feast, M. W., \& van Leeuwen, F. 2008, mnras, 386, 313

Groenewegen, M. A. T. 2008, A\&A, 488, 25 